\title{
Protein Aggregation Inhibitors as Disease-Modifying Therapies for Polyglutamine Diseases
}

\author{
Eiko N. Minakawa ${ }^{1 *}$ and Yoshitaka Nagai ${ }^{1,2 * t}$ \\ 'Department of Degenerative Neurological Diseases, National Institute of Neuroscience, National Center of Neurology \\ and Psychiatry, Kodaira, Japan, ${ }^{2}$ Department of Neurotherapeutics, Osaka University Graduate School of Medicine, Suita, \\ Japan
}

\section{OPEN ACCESS}

Edited by:

Ho Yin Edwin Chan,

The Chinese University of Hong Kong,

China

Reviewed by:

Yijuang Chern,

National Research Program for Biopharmaceuticals, Taiwan

Sung Bae Lee,

Daegu Gyeongbuk Institute of Science and Technology (DGIST),

South Korea

*Correspondence:

Eiko N. Minakawa

minakawa@ncnp.go.jp

Yoshitaka Nagai

yoshi.nagai@med.kindai.ac.jp

${ }^{\dagger}$ Present address:

Yoshitaka Nagai,

Department of Neurology, Kindai

University Faculty of Medicine,

Osaka-Sayama, Japan

Specialty section:

This article was submitted to

Neurodegeneration,

a section of the journal

Frontiers in Neuroscience

Received: 27 October 2020

Accepted: 18 January 2021

Published: 12 February 2021

Citation:

Minakawa EN and Nagai Y (2021)

Protein Aggregation Inhibitors as

Disease-Modifying Therapies

for Polyglutamine Diseases.

Front. Neurosci. 15:621996.

doi: 10.3389/fnins.2021.621996
The polyglutamine (polyQ) diseases are a group of inherited neurodegenerative diseases caused by the abnormal expansion of a CAG trinucleotide repeat that are translated into an expanded polyQ stretch in the disease-causative proteins. The expanded poly $Q$ stretch itself plays a critical disease-causative role in the pathomechanisms underlying polyQ diseases. Notably, the expanded polyQ stretch undergoes a conformational transition from the native monomer into the $\beta$-sheet-rich monomer, followed by the formation of soluble oligomers and then insoluble aggregates with amyloid fibrillar structures. The intermediate soluble species including the $\beta$-sheet-rich monomer and oligomers exhibit substantial neurotoxicity. Therefore, protein conformation stabilization and aggregation inhibition that target the upstream of the insoluble aggregate formation would be a promising approach toward the development of disease-modifying therapies for polyQ diseases. PolyQ aggregation inhibitors of different chemical categories, such as intrabodies, peptides, and small chemical compounds, have been identified through intensive screening methods. Among them, recent advances in the brain delivery methods of several peptides and the screening of small chemical compounds have brought them closer to clinical utility. Notably, the recent discovery of arginine as a potent conformation stabilizer and aggregation inhibitor of polyQ proteins both in vitro and in vivo have paved way to the clinical trial for the patients with poly $Q$ diseases. Meanwhile, expression reduction of expanded polyQ proteins per se would be another promising approach toward disease modification of polyQ diseases. Gene silencing, especially by antisense oligonucleotides (ASOs), have succeeded in reducing the expression of polyQ proteins in the animal models of various polyQ diseases by targeting the aberrant mRNA with expanded CAG repeats. Of note, some of these ASOs have recently been translated into clinical trials. Here we overview and discuss these recent advances toward the development of disease modifying therapies for polyQ diseases. We envision that combination therapies using aggregation inhibitors and gene silencing would meet the needs of the patients with polyQ diseases and their caregivers in the near future to delay or prevent the onset and progression of these currently intractable diseases.

Keywords: polyglutamine diseases, neurodegenerative diseases, aggregation inhibitor, protein misfolding, disease-modifying therapy, arginine 


\section{INTRODUCTION}

The polyglutamine (polyQ) diseases are a group of inherited neurodegenerative diseases that are caused by the abnormal expansion of a CAG triplet repeat (above $35-40$ repeats) in the coding region within the causative gene of each disease. This expanded CAG repeat is translated into an expanded polyQ stretch in the resultant protein product (Orr and Zoghbi, 2007). At least nine diseases including spinocerebellar ataxia (SCA) types 1, 2, 3, 6, 7, and 17, Huntington's disease (HD), spinal and bulbar muscular atrophy (SBMA), and dentatorubral pallidoluysian atrophy (DRPLA) are known so far to belong to this group of diseases (Table 1; Stoyas and La Spada, 2018).

The pathological hallmarks of polyQ diseases are the inclusion bodies that mainly consist of proteins with an expanded polyQ stretch and the progressive neuronal cell loss in the regions within the brains or spinal cords that are specific to each disease (Takeuchi and Nagai, 2017). Patients suffer from a variety of motor, cognitive, and psychiatric impairments that depends on the regions affected in the nervous system in each disease. Disease-modifying treatments that delay or halt the onset or progression of polyQ diseases remain an unmet clinical need (Nagai and Minakawa, 2015).

The causative genes of the nine polyQ diseases have neither sequence homology nor any functional similarities, except for the expanded CAG repeat that encodes an expanded polyQ stretch (Paulson, 2018). A wide variety of the cellular and molecular pathogenic events that are induced by these proteins with expanded polyQ stretch are largely shared by the nine different polyQ diseases (Stoyas and La Spada, 2018). Various studies using invertebrate and vertebrate animal models of polyQ diseases have shown that expanded polyQ stretch itself is sufficient to induce neuronal degeneration and leads to neurological impairment in vivo (Burright et al., 1995; Ikeda et al., 1996; Warrick et al., 1998; Faber et al., 1999). These findings indicate the critical disease-causative role of the expanded polyQ stretch in the pathogenesis of polyQ diseases.

Various in vitro structural studies including ours have shown that the expanded polyQ stretch undergoes a conformational transition from the native monomer into the $\beta$-sheet-rich monomer, followed by the formation of soluble oligomers and then insoluble aggregates with amyloid fibrillar structures (Figure 1; Chen et al., 2001, 2002; Masino et al., 2002; Poirier et al., 2002; Khare et al., 2005; Nagai et al., 2007; Nucifora et al., 2012). The presence of these intermediate soluble species preceding the formation of insoluble aggregates was confirmed in vivo using cultured cells (Takahashi et al., 2007, 2008; Olshina et al., 2010) and the brains of the HD (Legleiter et al., 2010; Sathasivam et al., 2010) and SBMA (Li et al., 2007) mice models. The presence of polyQ proteins with oligomer-like structure was also confirmed in the brains of patients with HD (Legleiter et al., 2010). Importantly, these intermediate soluble species exhibit significant neuronal toxicity (Kayed et al., 2003; Miller et al., 2011). Of note, we demonstrated that the monomeric conformer of the expanded polyQ protein with $\beta$-sheet-rich structure, as well as oligomers, exhibit cellular toxicity (Figure 1; Nagai et al., 2007). In contrast, $\alpha$-helical coiled-coil structure also has been demonstrated to contribute, at least in part, to the toxicity of polyQ proteins (Fiumara et al., 2010; Kwon et al., 2018). Of note, Fiumara et al. (2010) demonstrated that polyQ peptides themselves form $\alpha$-helical coiled-coil structure and assemble into oligomers, and those mutations that enhance coiled-coil propensity of polyQ proteins lead to increased aggregation and toxicity in cultured cells. The $\alpha$-helical coiled-coil structure of polyQ proteins were also prominently involved in proteinprotein interaction (Fiumara et al., 2010). In line with these findings, quantitative proteome of the insoluble fraction in HD mice model revealed that proteins sequestered with the polyQ aggregates were enriched with those containing coiledcoil structures (Hosp et al., 2017). In either case, formation of polyQ-positive aggregates or inclusion bodies per se does not correlate with neuronal cell death, and even decreases the risk of neuronal cell death, and hence may be a protective response of the cells against the intermediate soluble but toxic polyQ protein species (Klement et al., 1998; Saudou et al., 1998; Kuemmerle et al., 1999; Arrasate et al., 2004; Kim et al., 2016). Taken altogether, these findings indicate the significance of protein conformation stabilization and aggregation inhibition of the intermediate soluble species of expanded polyQ proteins toward the development of disease-modifying therapies for polyQ diseases (Figure 1; Takeuchi and Nagai, 2017).

\section{DISEASE-MODIFYING THERAPIES FOR POLYGLUTAMINE DISEASES VIA TARGETING CONFORMATION TRANSITION AND AGGREGATION OF EXPANDED POLYGLUTAMINE PROTEINS}

To achieve disease modification for polyQ diseases via conformation stabilization and aggregation inhibition of polyQ proteins, molecules of various categories have been screened using different approaches. Initial studies mainly searched for molecules such as intrabodies or peptides that directly bind to the expanded polyQ stretch. Such molecules are expected to exhibit anti-aggregation effect through alternation of the protein folding process because the protein folding kinetics are sensitive to the residues surrounding the protein itself (Robertson et al., 2011; Wetzel, 2012). In addition, such molecules may exhibit therapeutic effects by affecting the turnover or subcellular localization of the expanded polyQ proteins (Messer and Butler, 2020). On the other hand, various molecules including small chemical compounds have been screened using in vitro assays that directly test their anti-aggregation property. Molecules that were identified through such screenings are expected to exhibit therapeutic effect through direct or indirect molecular interaction with expanded polyQ proteins as discussed below.

\section{Intrabodies}

Intrabody is an antibody fragment that is bioactive inside cells and binds specifically to intracellular antigens (Messer and Butler, 2020). Since the identification of the first single-chain Fv (scFv) antibody, scFvC4, that specifically binds to the $\mathrm{N}$-terminal region 
TABLE 1 | The polyglutamine diseases.

\begin{tabular}{|c|c|c|c|}
\hline \multirow[t]{2}{*}{ Disease } & \multirow[t]{2}{*}{ Gene } & \multicolumn{2}{|c|}{ CAG repeat length } \\
\hline & & Normal & Disease \\
\hline $\begin{array}{l}\text { Spinal and bulbar } \\
\text { muscular atrophy (SBMA) }\end{array}$ & $\begin{array}{l}\text { Androgen receptor } \\
\text { (AR) }\end{array}$ & $9-36$ & $38-65$ \\
\hline Huntington's disease (HD) & Huntingtin (HTT) & $6-35$ & $36-180$ \\
\hline $\begin{array}{l}\text { Spinocerebeller ataxia } \\
\text { type } 1 \text { (SCA1) }\end{array}$ & Ataxin 1 (ATXN1) & $6-39$ & $39-83$ \\
\hline $\begin{array}{l}\text { Spinocerebeller ataxia } \\
\text { type } 2 \text { (SCA2) }\end{array}$ & Ataxin 2 (ATXN2) & $14-32$ & $32-200$ \\
\hline $\begin{array}{l}\text { Spinocerebeller ataxia } \\
\text { type } 3 \text { (SCA3) }\end{array}$ & Ataxin 3 (ATXN3) & $12-41$ & $55-84$ \\
\hline $\begin{array}{l}\text { Spinocerebeller ataxia } \\
\text { type } 6 \text { (SCA6) }\end{array}$ & $\begin{array}{l}\text { Calcium voltage-gated } \\
\text { channel subunit } \\
\text { alpha1 A (CACNA1A) }\end{array}$ & $4-19$ & $20-33$ \\
\hline $\begin{array}{l}\text { Spinocerebeller ataxia } \\
\text { type } 7 \text { (SCA7) }\end{array}$ & Ataxin 7 (ATXN7) & $4-35$ & $37-306$ \\
\hline $\begin{array}{l}\text { Spinocerebeller ataxia } \\
\text { type } 17 \text { (SCA17) }\end{array}$ & $\begin{array}{l}\text { TATA-box binding } \\
\text { protein (TBP) }\end{array}$ & $25-44$ & $46-63$ \\
\hline $\begin{array}{l}\text { Dentatorubral } \\
\text { pallidoluysian atrophy } \\
\text { (DRPLA) }\end{array}$ & Atrophin 1 (ATN1) & $6-36$ & $49-88$ \\
\hline
\end{tabular}

of HTT (Lecerf et al., 2001) and suppresses the formation of mutant HTT (mHTT)-positive aggregates in vivo (Wolfgang et al., 2005; Snyder-Keller et al., 2010), several intrabodies that inhibits mHTT aggregation and ameliorates the behavioral phenotypes of various HD animal models have been identified (Wang et al., 2008; Southwell et al., 2009, 2011; Amaro and Henderson, 2016).

The advantage of intrabodies for the treatment of HD is their high binding affinity to HTT protein, which could alter the misfolding process of mHTT protein as well as affect the turnover or subcellular localization of mHTT protein. On the contrary, when mHTT protein becomes fibrillar and insoluble, it can no longer be corrected by the intrabodies that have been developed so far (Messer and Butler, 2020). Another concern is that neither of the currently available intrabodies for HD do not exhibit longterm effect. In addition, the administration route of intrabodies to the brain is currently limited to gene delivery via viral vectors due to the large molecular size of intrabodies. Overcoming these limitations would bring intrabodies closer to clinical utility.

\section{Peptides and Small Chemical Compounds}

As discussed above, one of the critical issues to develop disease-modifying treatments for polyQ diseases is to enable repetitive and long-term delivery of the drugs to the brain. To overcome this issue, extensive screenings for low molecular weight compounds such as peptides and small chemical compounds that inhibit the aggregation of expanded polyQ proteins have been performed. So far, various peptides and small chemical compounds have been proven to exhibit antiaggregation propensity against expanded polyQ proteins through direct or indirect molecular interaction with expanded polyQ proteins. Among them, a number of peptides or small chemical

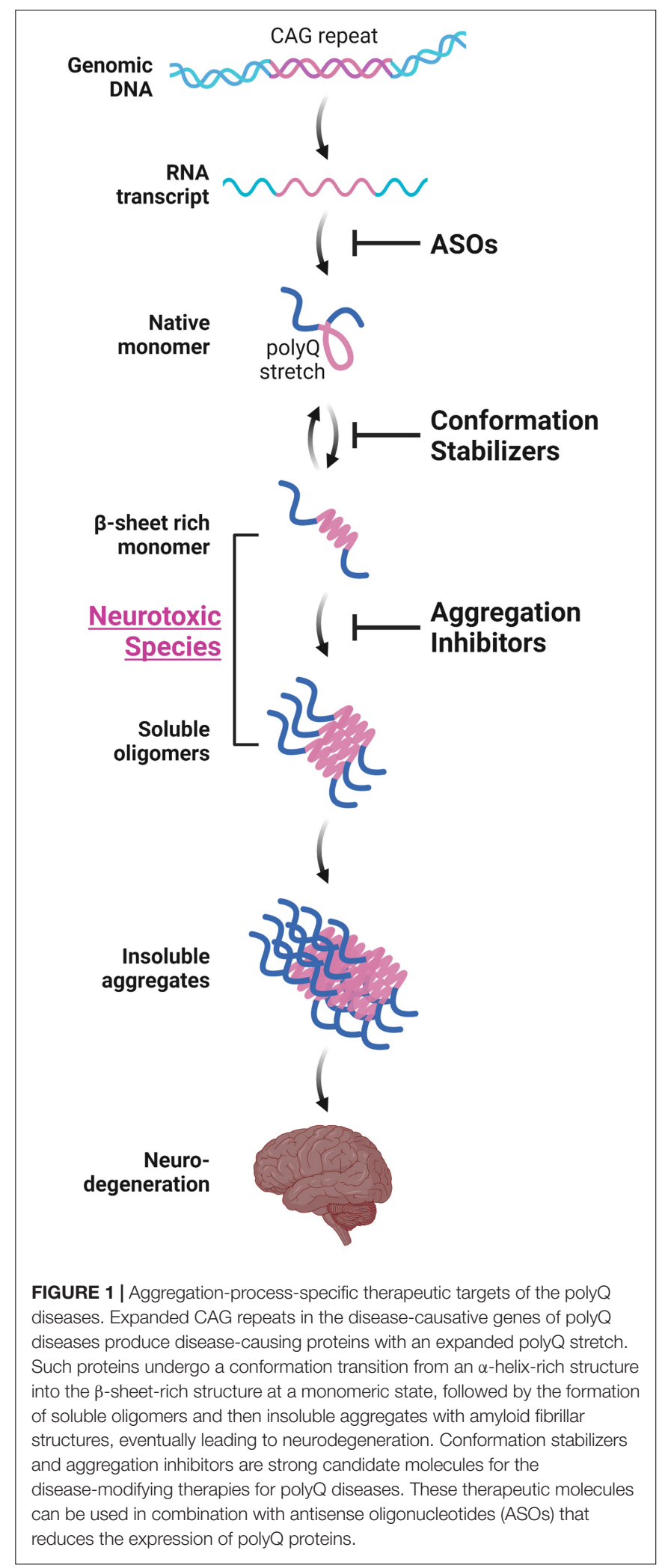

compounds have been demonstrated to exhibit neuroprotective effect in various in vivo models of polyQ diseases, as will be discussed in the following sections. As an alternative approach, 
various peptides and chemical compounds that exert therapeutic effects for polyQ disease models by activating the cellular protective mechanisms against expanded polyQ proteins, such as molecular chaperones, ubiquitin-proteasome system, and autophagy, have also been developed. In addition, several peptides and chemical compounds have also been developed that inhibit the downstream events such as aberrant calcium signaling or apoptosis evoked by expanded polyQ proteins and lead to cellular dysfunction or cell death. Peptides and chemical compounds targeting molecular processes other than the aggregation of polyQ proteins are beyond the scope of this article and have been recently reviewed elsewhere (Takeuchi et al., 2014; Takeuchi and Nagai, 2017).

\section{Peptides}

Peptides that specifically bind to expanded polyQ proteins but not to polyQ proteins with non-pathogenic length of polyQ stretch have been screened as aggregation inhibitor candidates of expanded polyQ proteins. Peptides that recognize and bind to this specific conformation of expanded polyQ proteins were expected to alter the kinetics of the protein misfolding and slow down or prevent the aggregation process, as was the case for intrabodies. This strategy was in accord with the identification of anti-polyQ monoclonal antibody1C2 that preferentially binds to polyQ proteins with longer polyQ stretch (Trottier et al., 1995), which implies the length-dependent difference in the tertiary structure of polyQ proteins.

We previously screened for peptides that selectively bind to the expanded polyQ stretch using a combinatorial peptide phage display libraries, and successfully identified several polyQbinding peptides including QBP1 (polyQ binding peptide 1; SNWKWWPGIFD) (Nagai et al., 2000). QBP1 exhibited a preferential high affinity for the expanded polyQ stretch with a dissociation constant (Kd) of $5.7 \mu \mathrm{M}$ (Okamoto et al., 2009). QBP1 inhibits the toxic $\beta$-sheet conformation transition of the polyQ protein monomer and its subsequent aggregation in vitro (Nagai et al., 2007). Further studies on its structureactivity relationship identified that the tryptophan-rich sequence is indispensable for the anti-aggregation property of QBP1 (Ren et al., 2001; Hamuro et al., 2007; Tomita et al., 2009) and that the original 11 amino acid sequence of QBP1 can be shortened to eight amino acids (WKWWPGIF) without losing its antiaggregation property against polyQ proteins. QBP1 expression in cultured cells (Nagai et al., 2000, 2007; Takahashi et al., 2007, 2008) or in Drosophila models of polyQ diseases (Nagai et al., 2003) significantly suppressed the formation of polyQ inclusions and polyQ-induced cell death. We also conjugated QBP1 with protein transduction domains (PTD-QBP1) to increase its membrane permeability, and showed that intracellular delivery of PTD-QBP1 successfully suppressed polyQ-induced premature death in Drosophila by oral administration of PTD-QBP1 (Popiel et al., 2007). Although we further examined the effect of PTDQBP1 on polyQ disease model mice, repeated intraperitoneal injection of PTD-QBP1 modestly improved only the body weight loss but not the motor phenotypes nor the inclusion formation in the brains (Popiel et al., 2009), probably due to insufficient delivery across the blood-brain barrier.
Recently, brain delivery methods of QBP1 were investigated. Intranasal administration of QBP1 using a thermosensitive gel with chitosan, an absorption enhancer for nasal delivery (Illum et al., 2001), was demonstrated to significantly elevate the concentration of QBP1 in rodents' brains for up to 10fold compared with intravenous administration or intranasal administration of water-dissolved QBP1 (Yang et al., 2018). In another recent study, biodegradable poly- $D, L$-lactide- $c 0$ glycolide (PLGA) nanoparticles encapsulating QBP1 was shown to inhibit polyQ protein aggregation in cultured neuronal cells and to suppress the motor dysfunction of Drosophila model of polyQ disease (Joshi et al., 2019). Considering that QBP1 was recently found to bind and exert anti-aggregation property not only to polyQ proteins but also to glutaminerich regions of the pathogenic amyloidogenic proteins such as TDP-43 (Mompeán et al., 2019), an aggregation-prone protein involved in the pathomechanisms of amyotrophic lateral sclerosis (ALS)/frontotemporal dementia (FTD), further studies are strongly awaited to increase the bioavailability of QBP1 itself or its derivatives or analogs.

Chen et al. (2011) also screened for potential polyQ aggregation inhibitors by a combinatorial screening for a library consisting of peptoids, a class of peptidomimetics comprised of $\mathrm{N}$-substituted glycine oligomers. Peptoids are resistant to degradation by protease and more permeable to cellular membranes compared with peptides (Simon et al., 1992). Through a screening of 60,000 diverse peptoids, they identified HQP09 as a specific ligand of expanded polyQ proteins (Chen et al., 2011). HQP09 suppressed polyQ aggregation in vitro, reduced polyQ-induced cell death in primary neuronal cultures, and decreased polyQ inclusion bodies in polyQ disease model mice by intracerebroventricular administration (Chen et al., 2011). Intriguingly, HQP09 and QBP1 did not compete with each other in binding expanded polyQ proteins, suggesting that HQP09 and QBP1 recognize the expanded polyQ stretch in nonoverlapping sites or structures (Chen et al., 2011). They further identified critical residues for HQP09 activity and generated HQP09_9, a minimal derivative of HQP09, that maintains the specificity in polyQ-binding property and polyQ aggregation inhibition property in vitro, and neuroprotective effects in primary neuronal cultures derived from polyQ disease model mice (Chen et al., 2011). Although subcutaneous administration of HQP09_9 did not exhibit therapeutic effect on polyQ disease model mice, HQP09_9 would serve as a lead compound to generate a novel polyQ aggregation inhibitor with better bioavailability.

Besides these screening approaches, recent studies have succeeded in designing aggregation inhibitor peptides with bipartite structures that consist of a domain with the ability to interact with aggregation-prone proteins and a domain with the ability to prevent their aggregation. He et al. (2020) designed a bipartite peptide, $8 \mathrm{R} 10 \mathrm{Q}$ that harbors a polyQ attached to a positively charged polyarginine. The polyQ sequence of $8 \mathrm{R} 10 \mathrm{Q}$ was expected to exhibit a specific and high affinity to the polyQ stretch in mHtt protein through its self-aggregating property, while the polyarginine sequence was expected to enable $8 \mathrm{R} 10 \mathrm{Q}$ to penetrate cell membrane, increase solubility and 
prevent self-aggregation of $8 \mathrm{R} 10 \mathrm{Q}$, and prevent the $\mathrm{mHtt} / 8 \mathrm{R} 10 \mathrm{Q}$ hybrid from self-aggregation. As expected, 8R10Q suppressed the oligomer and inclusion formation of mHTT proteins in cultured cells. In addition, intranasal administration of iodine-124-labeled $8 \mathrm{R} 10 \mathrm{Q}$ resulted in delivery of $8 \mathrm{RQ} 10$ to the brains, suppression of motor deficit, prolonged survival, and amelioration of mHTT aggregation and neuronal death in HD mouse model.

\section{Small Chemical Compounds}

Although various intrabodies, peptides, or peptoids that suppress the toxic conformation transition and aggregation of expanded polyQ proteins have been identified as discussed above, they are still of limited clinical use mainly due to poor drug delivery into mammalian brains. Screening for small chemical compounds that possess anti-aggregation property against expanded polyQ proteins both in vitro and in vivo, especially in mammalians, have therefore been intensively performed.

Since the first report by Wanker and colleagues (Heiser et al., 2002), various groups including ours have performed high-throughput screenings for polyQ aggregate inhibitors from large-scale chemical compound libraries using in vitro systems (Heiser et al., 2002; Tanaka et al., 2004; Ehrnhoefer et al., 2006) or cellular systems (Pollitt et al., 2003; Zhang et al., 2005; Fuentealba et al., 2012), although most of them have limitations for clinical application due to their toxicity, poor BBB permeability, or metabolic instability (Hockly et al., 2006; Frid et al., 2007). Several compounds among them have successfully exerted therapeutic effects in various in vivo models of polyQ diseases. For example, (-)-epigallo-catechin-3-gallate (EGCG), a green tea polyphenol, ameliorates neurodegeneration in a Drosophila model of $\mathrm{HD}$ (Ehrnhoefer et al., 2006) and a Caenorhabditis elegans model of SCA3 (Bonanomi et al., 2014). Trehalose, a disaccharide, delays the onset of neurological symptoms in a mouse model of $\mathrm{HD}$ (Tanaka et al., 2004). Y-27632, a Rho-activated protein kinase (ROCK) inhibitor, suppresses neurodegeneration in a Drosophila (Pollitt et al., 2003) and mouse model of HD (Li et al., 2009). HA-1077, a clinically approved ROCK inhibitor, also suppresses the polyQ-induced retinal degeneration when administered intravitreally in HD mouse model (Li et al., 2013). C2-8, a sulfobenzoic acid derivative, ameliorates neurodegeneration in a Drosophila model of HD (Zhang et al., 2005), although its effect in mice remains controversial despite its BBB permeability (Chopra et al., 2007; Wang et al., 2013).

Some of the small chemical compounds that exert antiaggregation property on other disease-causing proteins of neurodegenerative diseases, e.g., amyloid- $\beta$ (A $\beta)$, tau, prion, and $\alpha$-synuclein ( $\alpha$-Syn), also inhibits aggregation of polyQ proteins in vivo. For example, $\mathrm{N}^{\prime}$-benzylidenebenzohydrazide derivatives, an aggregation inhibitor of prion protein, suppresses polyQ protein aggregation in a zebrafish model of HD (Schiffer et al., 2007). Methylene blue, an aggregation inhibitor of tau, also suppresses polyQ protein aggregation and neurological phenotypes of the Drosophila and mouse models of HD (Sontag et al., 2012). Curcumin, an aggregation inhibitor of $A \beta, \alpha$-Syn, and prion protein, inhibits polyQ aggregation in yeast model (Verma et al., 2012) and knock-in mouse model (Hickey et al., 2012) of HD, although it did not ameliorate the motor deficit in the knock-in mouse model (Hickey et al., 2012). Intriguingly, a recent study showed that lipid membranes are a key modifier of the ability of small chemical compounds to inhibit polyQ aggregation (Beasley et al., 2019). While the polyQ aggregation inhibition property of curcumin was diminished under the presence of 1-palmitoyl-2-oleoyl-glycero-3-phosphocholine or total brain lipid extract vesicles, that of EGCG was not affected (Beasley et al., 2019). This study suggests the importance of taking into account the molecules surrounding the polyQ proteins in a crowded cellular environment in addition to the aggregation of polyQ protein perse.

Recently, we and others independently identified arginine as a potent aggregation inhibitor of polyQ proteins (Minakawa et al., 2020). Arginine belongs to a group of low molecular weight molecules named chemical chaperones. Chemical chaperones facilitate proper protein folding and suppress protein aggregation by stabilizing proteins in their native conformation and influencing the rate or fidelity of the protein-folding reaction (Welch and Brown, 1996; Cortez and Sim, 2014). Trehalose (Tanaka et al., 2004), proline (Ignatova and Gierasch, 2006), and cyclohexanol (McLaurin et al., 2006) are chemical chaperones that were previously shown to inhibit the aggregation of diseasecausing proteins including the polyQ proteins. Meanwhile, arginine has been well known to exhibit non-specific antiaggregation effect and is commonly used as an additive to prevent aggregation of various recombinant proteins expressed in Escherichia coli (Arakawa and Tsumoto, 2003). In addition, arginine has a high BBB permeability (Pardridge, 1983) and an established safety profile in humans since it is in clinical use for other human diseases such as urea cycle deficits and mitochondrial myopathy, encephalopathy, lactic acid, and stroke (MELAS) syndrome (Koga et al., 2002; Boenzi et al., 2012; Koenig et al., 2016). Although there are reports of diarrhea with varying incidence following oral administration of arginine especially at single high-dose administration possibly due to arginine-induced water and electrolyte secretion (Grimble, 2007), long-term oral administration of relatively high-dose arginine $(0.3-0.5 \mathrm{~g} / \mathrm{kg} / \mathrm{day}$ orally in three divided doses after each meal for 2 years) is reported to be well tolerable with no severe adverse effect related with arginine (Koga et al., 2018).

We identified arginine as a potent polyQ aggregation inhibitor by screening a library of representative chemical chaperones using an in vitro polyQ aggregation assay (Minakawa et al., 2020). Importantly, arginine suppresses the most upstream process of polyQ protein aggregation, which is the toxic conformational transition of polyQ proteins from an $\alpha$-helix-rich monomer to a toxic $\beta$-sheet-rich monomer, and also the downstream process such as the oligomer formation, aggregation formation, and seedinduced aggregation of polyQ proteins (Minakawa et al., 2020). Oral administration of arginine suppresses motor deficit, polyQ aggregation pathology, and neurodegeneration of multiple in vivo invertebrate and vertebrate models of polyQ diseases including two different mice models of SCA1 and SBMA (Minakawa et al., 2020). Of note, arginine exerted therapeutic effect on the motor deficit of SCA1 model mice even when administered after symptom onset (Minakawa et al., 2020). Based on these results and the established safety of arginine in humans, we have 
scheduled a clinical trial to evaluate the efficacy and safety of long-term arginine administration in polyQ patients in Japan.

In a recent independent study, Singh et al. (2019) showed that arginine and arginine ethyl ester (AEE) suppresses the aggregation of HTT exon 1 with an expanded polyQ stretch (mHTTex1) and rescues the motor deficit of the Drosophila HD model. Arginine consists of a guanidino group in the N-terminus and a glycine group in the C-terminus. They identified that the guanidino group of arginine is necessary for its anti-aggregation propensity against mHTTex1. Meanwhile, AEE and arginine methyl ester showed stronger anti-aggregation propensity than arginine (Singh et al., 2019). Arginine does not directly bind to mHTTex1, but instead alters the hydrogen bonding network (Singh et al., 2019). In contrast, AEE directly binds to mHTTex1 at the $\mathrm{N}$-terminal domain in addition to altering the hydrogen bonding network (Singh et al., 2019). Consistently, AEE exhibited a higher anti-aggregation effect on mHTTex1, and a better rescue of the motor deficit of Drosophila HD model compared with arginine (Singh et al., 2019).

These recent two studies suggest that arginine is a promising candidate molecule for the disease-modifying therapy of polyQ diseases, although it should be noted that a slight but significant elevation of plasma arginine level is reported in an animal model of HD (Skene et al., 2017) and that it is not still conclusive whether this elevation is protective or pathogenic for HD. Meanwhile, the non-specific anti-aggregation property of arginine would enable the use of arginine for the treatment of neurodegenerative disease in common, since protein misfolding is the common pathomechanism underlying neurodegenerative diseases such as Alzheimer's disease (AD) or Parkinson's disease (PD). Indeed, arginine suppresses the aggregation of $\mathrm{A} \beta$ or $\alpha$-Syn in vitro (Das et al., 2007; Ghosh et al., 2018), both of which aggregate, exhibit neurotoxicity, and accumulate in the brains of patients with $\mathrm{AD}$ or PD. Alternatively, arginine derivatives with modification in the glycine group but not the guanidino group would help increase the specificity and efficacy of arginine as a polyQ aggregation inhibitor. Meanwhile, as we previously discussed, formation of polyQ-positive aggregates may be a protective response of the cells against the intermediate soluble but toxic protein species. From this point of view, it should be noted that the aforementioned aggregation inhibitors, not limited to arginine and its derivatives, might have exhibited neuroprotective effects through their ability to modulate the toxicity of these proteins, e.g., by altering protein interaction (Kim et al., 2016), in addition to their in vitro ability to modulate the aggregation process.

\section{DISEASE-MODIFYING THERAPIES FOR POLYGLUTAMINE DISEASES VIA REDUCTION OF TOXIC POLYGLUTAMINE PROTEIN EXPRESSION USING ANTISENSE OLIGONUCLEOTIDES}

Besides the aggregation inhibition of polyQ proteins by various aforementioned methods, reducing the expression of expanded
polyQ proteins per se is an attractive approach toward the development of disease-modification therapy for polyQ diseases. Among the preclinical studies using RNA interference (RNAi)based methods or antisense oligonucleotides (ASOs), both of which alter the polyQ protein expression by targeting the aberrant mRNA with expanded CAG repeats, ASOs for the treatment of $\mathrm{HD}$ have recently been translated into clinical trials. In addition to the therapeutic effects of ASOs, the safety, tolerability, and effective delivery of ASOs in humans have been established in clinical studies for spinal muscular atrophy or Duchenne muscular dystrophy, and lead to the recent approval of the ASOs such as nusinersen or eteplirsen, respectively, by the Food and Drug Administration in the United States (Schoch and Miller, 2017). ASOs therefore are promising candidates for the disease-modifying therapies for polyQ diseases which could be used in combination with aggregation inhibitors (Figure 1).

Antisense oligonucleotides are synthetic single-stranded oligonucleotides that bind to RNA molecules. ASOs can be designed in an allele-specific or non-allele-specific manner to reduce the expression of target protein either by degrading the mRNA via recruitment of RNase $\mathrm{H}$, inducing exon skipping by regulating the splicing machinery at specific exon-intron junctions, or inhibiting protein translation by interfering with the translational machinery (Bennett and Swayze, 2010). Initial preclinical studies utilizing ASOs for the treatment of polyQ diseases took allele-specific approaches and targeted either the expanded CAG repeat in the mRNA (Hu et al., 2009) or single nucleotide polymorphisms (SNPs) that are associated with the disease (Carroll et al., 2011). Allele-specific ASOs targeting the mutated gene would be favorable when the protein product from wild-type mRNA is physiologically indispensable. However, this approach might not be applicable to patients homozygous for disease-causing mutations or disease-related SNPs. In addition, especially in polyQ diseases, allele-specific ASOs targeting CAG repeats in the mutant allele may result in the reduced expression of physiologically indispensable proteins because the wild-type allele of the disease-causing genes and genes of various other proteins also contain CAG repeats, although chemical modification of ASOs may help overcome this limitation (Rué et al., 2016). Furthermore, multiple allele-specific ASOs for a single disease need to be developed when multiple SNPs are associated with the disease, as is the case with polyQ diseases. Accordingly, most preclinical studies have utilized non-allele specific ASOs or allele-specific ASOs for disease-related SNPs associated with polyQ diseases.

Non-allele specific ASO targeting ATXN1 significantly reduced the expression of ATXN1, ameliorated the motor deficit, and prolonged the survival of SCA1 knock-in mice by intracerebroventricular (ICV) administration (Friedrich et al., 2018). Similarly, ICV administration of non-allele specific ASO targeting ATXN2 significantly reduced the expression of ATXN2 and ameliorated the motor deficit in two different mice models of SCA2 (Scoles et al., 2017). Of note, this ASO restored the firing rate of Purkinje cells even when administered after the motor phenotype onset of the SCA2 
knock-in mice (Scoles et al., 2017). ICV administration of non-allele specific ASO targeting ATXN3 also significantly reduced the expression of $\mathrm{ATXN} 3$, ameliorated the motor deficit, and restored the firing rate of Purkinje cells (McLoughlin et al., 2018). Importantly, this ASO successfully reduced the high molecular weight ATXN3 aggregates and prevented the nuclear localization of ATXN3 in two different mice models of SCA3 (Moore et al., 2017; McLoughlin et al., 2018). Non-allele specific ASOs for SCA7 (Niu et al., 2018), HD (Kordasiewicz et al., 2012; Stanek et al., 2013), and SBMA (Lieberman et al., 2014; Sahashi et al., 2015), and allele-specific ASOs for HD (Sun et al., 2014; Rué et al., 2016; Datson et al., 2017; Southwell et al., 2018) that are effective in the mice or non-human-primate model of each disease have also been identified.

Based on these studies, the first phase $1 / 2$ clinical trial was performed using a non-allele-specific ASO in patients with HD (Tabrizi et al., 2019). This ASO showed good tolerability without serious adverse effects, and induced a dose-dependent reduction of mHTT in the cerebrospinal fluid (Tabrizi et al., 2019). An open-labeled extension study for patients with $\mathrm{HD}$ who completed the phase $1 / 2$ trial (NCT03342053) and a phase 3 trial (NCT03761849) using the same ASO is ongoing. In addition, two other phase $1 / 2$ trials (NCT03225833 and NCT03225846) are also ongoing, which uses allele-specific ASOs that target mutant-alleleassociated SNPs.

The advantages of ASOs in the treatment of polyQ diseases are their high specificity and established safety, which have led to successful translation of preclinical to clinical studies in $\mathrm{HD}$ as discussed above. Meanwhile, although ASOs apparently distributes widely throughout the brain by intrathecal or ICV administration in rodents and non-human primates, ASO concentration may be lower in deeper brain regions such as the striatum (Kordasiewicz et al., 2012). Although intrathecal delivery of ASOs showed therapeutic effects in the clinical trials for SMA, this might have been because SMA mainly affects the spinal cord. In addition, currently available ASOs needs repeated administration to maintain the therapeutic effect. Development of better ASO administration methods that ensure prolonged delivery to the target brain regions and are less invasive is strongly awaited. In addition, the extremely high cost of the FDA-approved ASOs remains a challenge in clinical settings (Burgart et al., 2018).

\section{CONCLUSION}

Disease-modifying treatment for polyQ diseases have long been an unmet clinical need. Although various chemical compounds have been demonstrated to inhibit the aggregation of polyQ proteins in vitro, most of them were of limited use in the clinical settings due to their toxicity, poor BBB permeability, or metabolic instability. Among the aggregation inhibitors of polyQ proteins, recent studies have identified promising candidate therapeutic molecules that is effective in vivo and could achieve prompt clinical application. One of such molecules is arginine, which already has an established safety in humans and fits the recent concept of "drug repositioning” (Corbett et al., 2012). Notably, arginine was effective in polyQ disease model mice even after symptom onset. Meanwhile, great efforts are under way to achieve better drug delivery of potent aggregation inhibitor peptides, peptoids or intrabodies. Together with the recent success of ASOs in the clinical trials to reduce the expression of polyQ proteins in patients with $\mathrm{HD}$, combination therapies using gene silencing and aggregation inhibitors for the remaining polyQ proteins would enhance the possibility for delaying or halting the onset and progression of polyQ diseases in the near future.

\section{AUTHOR CONTRIBUTIONS}

EM and YN designed the manuscript and assessed the literature. EM wrote the initial draft of the manuscript. EM and YN edited the subsequent drafts and revisions. Both authors contributed to the article and approved the submitted version.

\section{FUNDING}

This work was funded by Grants-in-Aid for Scientific Research on Priority Areas (Advanced Brain Science Project 16015275, Research on Pathomechanisms of Brain Disorders 20023019, and Protein Community 22020038 to YN) and on Innovative Areas (Synapse and Neurocircuit Pathology 25110741 and Brain Protein Aging and Dementia Control 17H05699 to YN) from the Ministry of Education, Culture, Sports, Science, and Technology, Japan; by Grants-in-Aid for Scientific Research (B) (20390245 to YN) and for Challenging Exploratory Research (22659172 to YN) from the Japan Society for the Promotion of Science (JSPS), Japan; by Health Labor Sciences Research Grants for Research on Development of New Drugs (H24-Soyaku-Sogo002 to YN), Research for Intractable Diseases (H26-Nanchi025 to YN), Research for Persons with Disabilities (H25Shinkei-Kin-003 to YN), and the Research Committee for Ataxic Diseases (H23-Nanchi-014 to YN) from the Ministry of Health, Labor and Welfare, Japan; by grants for Practical Research Projects for Rare/Intractable Diseases (JP16ek0109018, 16ek0109048, JP19ek0109222, and JP20ek0109459 to YN) and for Project of Translational and Clinical Research Core Centers (JP17lm0203035 and JP18lm0203071 to YN) from the Japan Agency for Medical Research and Development; by a grant for Core Research for Evolutional Science and Technology (CREST) (to YN) from the Japan Science and Technology Agency; and by a grant from the Takeda Science Foundation (to YN).

\section{ACKNOWLEDGMENTS}

The figure was created with the support of BioRender.com. 


\section{REFERENCES}

Amaro, I. A., and Henderson, L. A. (2016). An intrabody drug (rAAV6-INT41) reduces the binding of $\mathrm{N}$-terminal Huntingtin fragment(s) to DNA to basal levels in PC12 cells and delays cognitive loss in the R6/2 animal model. J. Neurodegener. Dis. 2016:7120753.

Arakawa, T., and Tsumoto, K. (2003). The effects of arginine on refolding of aggregated proteins: not facilitate refolding, but suppress aggregation. Biochem. Biophys. Res. Commun. 304, 148-152. doi: 10.1016/s0006-291x(03) 00578-3

Arrasate, M., Mitra, S., Schweitzer, E. S., Segal, M. R., and Finkbeiner, S. (2004). Inclusion body formation reduces levels of mutant huntingtin and the risk of neuronal death. Nature 431, 805-810. doi: 10.1038/nature02998

Beasley, M., Stonebraker, A. R., Hasan, I., Kapp, K. L., Liang, B. J., Agarwal, G., et al. (2019). Lipid membranes influence the ability of small molecules to inhibit Huntingtin fibrillization. Biochemistry 58, 4361-4373. doi: 10.1021/acs. biochem.9b00739

Bennett, C. F., and Swayze, E. E. (2010). RNA targeting therapeutics: molecular mechanisms of antisense oligonucleotides as a therapeutic platform. Аnnu. Rev. Pharmacol. Toxicol. 50, 259-293. doi: 10.1146/annurev.pharmtox.010909. 105654

Boenzi, S., Pastore, A., Martinelli, D., Goffredo, B. M., Boiani, A., Rizzo, C., et al. (2012). Creatine metabolism in urea cycle defects. J. Inherit Metab. Dis. 35, 647-653.

Bonanomi, M., Natalello, A., Visentin, C., Pastori, V., Penco, A., Cornelli, G., et al. (2014). Epigallocatechin-3-gallate and tetracycline differently affect ataxin-3 fibrillogenesis and reduce toxicity in spinocerebellar ataxia type 3 model. Hum. Mol. Genet. 23, 6542-6552. doi: 10.1093/hmg/ddu373

Burgart, A. M., Magnus, D., Tabor, H. K., Paquette, E. D.-T., Frader, J., Glover, J. J., et al. (2018). Ethical challenges confronted when providing Nusinersen treatment for spinal muscular atrophy. JAMA Pediatr. 172, 188-192. doi: 10.1001/jamapediatrics.2017.4409

Burright, E. N., Brent Clark, H., Servadio, A., Matilla, T., Feddersen, R. M., Yunis, W. S., et al. (1995). SCA1 transgenic mice: a model for neurodegeneration caused by an expanded CAG trinucleotide repeat. Cell 82, 937-948. doi: 10. 1016/0092-8674(95)90273-2

Carroll, J. B., Warby, S. C., Southwell, A. L., Doty, C. N., Greenlee, S., Skotte, N., et al. (2011). Potent and selective antisense oligonucleotides targeting singlenucleotide polymorphisms in the Huntington disease gene / allele-specific silencing of mutant Huntingtin. Mol. Ther. 19, 2178-2185. doi: 10.1038/mt. 2011.201

Chen, S., Berthelier, V., Hamilton, J. B., O’Nuallai, B., and Wetzel, R. (2002). Amyloid-like features of polyglutamine aggregates and their assembly kinetics. Biochemistry 41, 7391-7399. doi: 10.1021/bi011772q

Chen, S., Berthelier, V., Yang, W., and Wetzel, R. (2001). Polyglutamine aggregation behavior in vitro supports a recruitment mechanism of cytotoxicity. J. Mol. Biol. 311, 173-182. doi: 10.1006/jmbi.2001.4850

Chen, X., Wu, J., Luo, Y., Liang, X., Supnet, C., Kim, M. W., et al. (2011). Expanded Polyglutamine-binding peptoid as a novel therapeutic agent for treatment of Huntington's disease. Chem. Biol. 18, 1113-1125. doi: 10.1016/j.chembiol.2011. 06.010

Chopra, V., Fox, J. H., Lieberman, G., Dorsey, K., Matson, W., Waldmeier, P., et al. (2007). A small-molecule therapeutic lead for Huntington's disease: preclinical pharmacology and efficacy of C2-8 in the R6/2 transgenic mouse. Proc. Natl. Acad. Sci. U.S.A. 104, 16685-16689. doi: 10.1073/pnas.0707842104

Corbett, A., Pickett, J., Burns, A., Corcoran, J., Dunnett, S. B., Edison, P., et al. (2012). Drug repositioning for Alzheimer's disease. Nat. Rev. Drug Discov. 11, 833-846.

Cortez, L., and Sim, V. (2014). The therapeutic potential of chemical chaperones in protein folding diseases. Prion 8, 197-202. doi: 10.4161/pri.28938

Das, U., Hariprasad, G., Ethayathulla, A. S., Manral, P., Das, T. K., Pasha, S., et al. (2007). Inhibition of protein aggregation: supramolecular assemblies of arginine hold the key. PLOS ONE 2:e1176. doi: 10.1371/journal.pone.00 01176

Datson, N. A., González-Barriga, A., Kourkouta, E., Weij, R., van de Giessen, J., Mulders, S., et al. (2017). The expanded CAG repeat in the huntingtin gene as target for therapeutic RNA modulation throughout the HD mouse brain. PLoS One 12:e0171127. doi: 10.1371/journal.pone.0171127
Ehrnhoefer, D. E., Duennwald, M., Markovic, P., Wacker, J. L., Engemann, S., Roark, M., et al. (2006). Green tea (-)-epigallocatechin-gallate modulates early events in huntingtin misfolding and reduces toxicity in Huntington's disease models. Hum. Mol. Genet. 15, 2743-2751. doi: 10.1093/hmg/ddl210

Faber, P. W., Alter, J. R., MacDonald, M. E., and Hart, A. C. (1999). Polyglutaminemediated dysfunction and apoptotic death of a Caenorhabditis elegans sensory neuron. PNAS 96, 179-184. doi: 10.1073/pnas.96.1.179

Fiumara, F., Fioriti, L., Kandel, E. R., and Hendrickson, W. A. (2010). Essential role of coiled coils for aggregation and activity of Q/N-Rich prions and PolyQ Proteins. Cell 143, 1121-1135. doi: 10.1016/j.cell.2010.11.042

Frid, P., Anisimov, S. V., and Popovic, N. (2007). Congo red and protein aggregation in neurodegenerative diseases. Brain Res. Rev. 53, 135-160. doi: 10.1016/j.brainresrev.2006.08.001

Friedrich, J., Kordasiewicz, H. B., O'Callaghan, B., Handler, H. P., Wagener, C., Duvick, L., et al. (2018). Antisense oligonucleotide-mediated ataxin-1 reduction prolongs survival in SCA1 mice and reveals disease-associated transcriptome profiles. JCI Insight 3:e123193.

Fuentealba, R. A., Marasa, J., Diamond, M. I., Piwnica-Worms, D., and Weihl, C. C. (2012). An aggregation sensing reporter identifies leflunomide and teriflunomide as polyglutamine aggregate inhibitors. Hum. Mol. Genet. 21, 664-680. doi: $10.1093 / \mathrm{hmg} / \mathrm{ddr} 500$

Ghosh, S., Kundu, A., and Chattopadhyay, K. (2018). Small molecules attenuate the interplay between conformational fluctuations, early oligomerization and amyloidosis of alpha synuclein. Sci. Rep. 8:5481.

Grimble, G. K. (2007). Adverse gastrointestinal effects of arginine and related amino acids. J. Nutr. 137, 1693S-1701S.

Hamuro, L., Zhang, G., Tucker, T. J., Self, C., Strittmatter, W. J., and Burke, J. R. (2007). Optimization of a polyglutamine aggregation inhibitor peptide (QBP1) using a thioflavin T fluorescence assay. ASSAY Drug Dev. Technol. 5, 629-636. doi: $10.1089 /$ adt.2007.083

He, R.-Y., Lai, X.-M., Sun, C.-S., Kung, T.-S., Hong, J.-Y., Jheng, Y.-S., et al. (2020). Nanoscopic insights of amphiphilic peptide against the oligomer assembly process to treat Huntington's disease. Adv. Sci. 7:1901165. doi: 10.1002/advs. 201901165

Heiser, V., Engemann, S., Bröcker, W., Dunkel, I., Boeddrich, A., Waelter, S., et al. (2002). Identification of benzothiazoles as potential polyglutamine aggregation inhibitors of Huntington's disease by using an automated filter retardation assay. Proc. Natl. Acad. Sci. U.S.A. 99(Suppl. 4), 16400-16406. doi: 10.1073/ pnas. 182426599

Hickey, M. A., Zhu, C., Medvedeva, V., Lerner, R. P., Patassini, S., Franich, N. R., et al. (2012). Improvement of neuropathology and transcriptional deficits in CAG 140 knock-in mice supports a beneficial effect of dietary curcumin in Huntington's disease. Mol. Neurodegener. 7:12. doi: 10.1186/1750-13 26-7-12

Hockly, E., Tse, J., Barker, A. L., Moolman, D. L., Beunard, J.-L., Revington, A. P., et al. (2006). Evaluation of the benzothiazole aggregation inhibitors riluzole and PGL-135 as therapeutics for Huntington's disease. Neurobiol. Dis. 21, 228-236. doi: 10.1016/j.nbd.2005.07.007

Hosp, F., Gutiérrez-Ángel, S., Schaefer, M. H., Cox, J., Meissner, F., Hipp, M. S., et al. (2017). Spatiotemporal proteomic profiling of Huntington's disease inclusions reveals widespread loss of protein function. Cell Rep. 21, 2291-2303. doi: 10.1016/j.celrep.2017.10.097

Hu, J., Matsui, M., Gagnon, K. T., Schwartz, J. C., Gabillet, S., Arar, K., et al. (2009). Inhibiting expression of mutant huntingtin and ataxin-3 by targeting expanded CAG repeat RNAs. Nat. Biotechnol. 27, 478-484. doi: 10.1038/nbt.1539

Ignatova, Z., and Gierasch, L. M. (2006). Inhibition of protein aggregation in vitro and in vivo by a natural osmoprotectant. Proc. Natl. Acad. Sci. U.S.A. 103, 13357-13361. doi: 10.1073/pnas.0603772103

Ikeda, H., Yamaguchi, M., Sugai, S., Aze, Y., Narumiya, S., and Kakizuka, A. (1996). Expanded polyglutamine in the Machado-Joseph disease protein induces cell death in vitro and in vivo. Nat. Genet. 13, 196-202. doi: 10.1038/ng06 96-196

Illum, L., Jabbal-Gill, I., Hinchcliffe, M., Fisher, A. N., and Davis, S. S. (2001). Chitosan as a novel nasal delivery system for vaccines. Adv. Drug Deliv. Rev. 51, 81-96. doi: 10.1016/s0169-409x(01)00171-5

Joshi, A. S., Singh, V., Gahane, A., and Thakur, A. K. (2019). biodegradable nanoparticles containing mechanism based peptide inhibitors reduce polyglutamine aggregation in cell models and alleviate motor symptoms 
in a Drosophila model of Huntington's disease. ACS Chem. Neurosci. 10, 1603-1614. doi: 10.1021/acschemneuro.8b00545

Kayed, R., Head, E., Thompson, J. L., McIntire, T. M., Milton, S. C., Cotman, C. W., et al. (2003). Common structure of soluble amyloid oligomers implies common mechanism of pathogenesis. Science 300, 486-489. doi: 10.1126/ science. 1079469

Khare, S. D., Ding, F., Gwanmesia, K. N., and Dokholyan, N. V. (2005). Molecular origin of polyglutamine aggregation in neurodegenerative diseases. PLoS Comput. Biol. 1:e30. doi: 10.1371/journal.pcbi.0010030

Kim, Y. E., Hosp, F., Frottin, F., Ge, H., Mann, M., Hayer-Hartl, M., et al. (2016). Soluble oligomers of PolyQ-expanded Huntingtin target a multiplicity of key cellular factors. Mol. Cell 63, 951-964. doi: 10.1016/j.molcel.2016.07.022

Klement, I. A., Skinner, P. J., Kaytor, M. D., Yi, H., Hersch, S. M., Clark, H. B., et al. (1998). Ataxin-1 nuclear localization and Aggregation: role in polyglutamineinduced disease in SCA1 transgenic mice. Cell 95, 41-53.

Koenig, M. K., Emrick, L., Karaa, A., Korson, M., Scaglia, F., Parikh, S., et al. (2016). Recommendations for the management of strokelike episodes in patients with mitochondrial encephalomyopathy, lactic acidosis, and strokelike episodes. JAMA Neurol. 73, 591-594. doi: 10.1001/jamaneurol.2015.5072

Koga, Y., Ishibashi, M., Ueki, I., Yatsuga, S., Fukiyama, R., Akita, Y., et al. (2002). Effects of L-arginine on the acute phase of strokes in three patients with MELAS. Neurology 58, 827-828. doi: 10.1212/wnl.58.5.827

Koga, Y., Povalko, N., Inoue, E., Nakamura, H., Ishii, A., Suzuki, Y., et al. (2018). Therapeutic regimen of 1-arginine for MELAS: 9-year, prospective, multicenter, clinical research. J. Neurol. 265, 2861-2874. doi: 10.1007/s00415-0189057-7

Kordasiewicz, H. B., Stanek, L. M., Wancewicz, E. V., Mazur, C., McAlonis, M. M., Pytel, K. A., et al. (2012). Sustained therapeutic reversal of Huntington's disease by transient repression of Huntingtin synthesis. Neuron 74, 1031-1044. doi: 10.1016/j.neuron.2012.05.009

Kuemmerle, S., Gutekunst, C.-A., Klein, A. M., Li, X.-J., Li, S.-H., Beal, M. F., et al. (1999). Huntingtin aggregates may not predict neuronal death in Huntington's disease. Ann. Neurol. 46, 842-849. doi: 10.1002/1531-8249(199912)46:6<842:: aid-ana6 $>3.0 .00 ; 2-o$

Kwon, M. J., Han, M. H., Bagley, J. A., Hyeon, D. Y., Ko, B. S., Lee, Y. M., et al. (2018). Coiled-coil structure-dependent interactions between polyQ proteins and Foxo lead to dendrite pathology and behavioral defects. PNAS 115, E10748E10757.

Lecerf, J.-M., Shirley, T. L., Zhu, Q., Kazantsev, A., Amersdorfer, P., Housman, D. E., et al. (2001). Human single-chain Fv intrabodies counteract in situ huntingtin aggregation in cellular models of Huntington's disease. PNAS 98, 4764-4769. doi: 10.1073/pnas.071058398

Legleiter, J., Mitchell, E., Lotz, G. P., Sapp, E., Ng, C., DiFiglia, M., et al. (2010). Mutant huntingtin fragments form oligomers in a polyglutamine lengthdependent manner in vitro and in vivo. J. Biol. Chem. 285, 14777-14790. doi: 10.1074/jbc.m109.093708

Li, M., Chevalier-Larsen, E. S., and Merry, D. E. (2007). Diamond MI. soluble androgen receptor oligomers underlie pathology in a mouse model of spinobulbar muscular atrophy. J. Biol. Chem. 282, 3157-3164. doi: 10.1074/ jbc.m609972200

Li, M., Huang, Y., Ma, A. A. K., Lin, E., and Diamond, M. I. (2009). Y-27632 improves rotarod performance and reduces huntingtin levels in R6/2 mice. Neurobiol. Dis. 36, 413-420. doi: 10.1016/j.nbd.2009.06.011

Li, M., Yasumura, D., Ma, A. A. K., Matthes, M. T., Yang, H., Nielson, G., et al. (2013). Intravitreal administration of HA-1077, a ROCK inhibitor, improves retinal function in a mouse model of Huntington disease. PLoS One 8:e56026. doi: 10.1371/journal.pone. 0056026

Lieberman, A. P., Yu, Z., Murray, S., Peralta, R., Low, A., Guo, S., et al. (2014). Peripheral Androgen Receptor Gene Suppression Rescues Disease in Mouse Models of Spinal and Bulbar Muscular Atrophy. Cell Reports 7, 774-784. doi: 10.1016/j.celrep.2014.02.008

Masino, L., Kelly, G., Leonard, K., Trottier, Y., and Pastore, A. (2002). Solution structure of polyglutamine tracts in GST-polyglutamine fusion proteins. FEBS Lett. 513, 267-272. doi: 10.1016/s0014-5793(02)02335-9

McLaurin, J., Kierstead, M. E., Brown, M. E., Hawkes, C. A., Lambermon, M. H. L., Phinney, A. L., et al. (2006). Cyclohexanehexol inhibitors of Abeta aggregation prevent and reverse Alzheimer phenotype in a mouse model. Nat. Med. 12, 801-808. doi: $10.1038 / \mathrm{nm} 1423$
McLoughlin, H. S., Moore, L. R., Chopra, R., Komlo, R., McKenzie, M., Blumenstein, K. G., et al. (2018). Oligonucleotide therapy mitigates disease in Spinocerebellar ataxia type 3 mice. Ann. Neurol. 84, 64-77. doi: 10.1002/ana. 25264

Messer, A., and Butler, D. C. (2020). Optimizing intracellular antibodies (intrabodies/nanobodies) to treat neurodegenerative disorders. Neurobiol. Dis. 134:104619. doi: 10.1016/j.nbd.2019.104619

Miller, J., Arrasate, M., Brooks, E., Peters-Libeu, C., Legleiter, J., Hatters, D., et al. (2011). Identifying polyglutamine protein species in situ that best predict neurodegeneration. Nat. Chem. Biol. 7, 925-934. doi: 10.1038/nchembio.694

Minakawa, E. N., Popiel, H. A., Tada, M., Takahashi, T., Yamane, H., Saitoh, Y., et al. (2020). Arginine is a disease modifier for polyQ disease models that stabilizes polyQ protein conformation. Brain 143, 1811-1825. doi: 10.1093/ brain/awaal15

Mompeán, M., Ramírez de Mingo, D., Hervás, R., Fernández-Ramírez, M., del, C., Carrión-Vázquez, M., et al. (2019). Molecular mechanism of the inhibition of TDP-43 amyloidogenesis by QBP1. Arch. Biochem. Biophys. 675:108113. doi: 10.1016/j.abb.2019.108113

Moore, L. R., Rajpal, G., Dillingham, I. T., Qutob, M., Blumenstein, K. G., Gattis, D., et al. (2017). Evaluation of antisense oligonucleotides targeting ATXN3 in SCA3 mouse models. Mol. Ther. Nucleic Acids 7, 200-210. doi: 10.1016/j.omtn. 2017.04.005

Nagai, Y., Fujikake, N., Ohno, K., Higashiyama, H., Popiel, H. A., Rahadian, J., et al. (2003). Prevention of polyglutamine oligomerization and neurodegeneration by the peptide inhibitor QBP1 in Drosophila. Hum. Mol. Genet. 12, 1253-1259. doi: $10.1093 / \mathrm{hmg} / \mathrm{ddg} 144$

Nagai, Y., Inui, T., Popiel, H. A., Fujikake, N., Hasegawa, K., Urade, Y., et al. (2007). A toxic monomeric conformer of the polyglutamine protein. Nat. Struct. Mol. Biol. 14, 332-340. doi: 10.1038/nsmb1215

Nagai, Y., and Minakawa, E. N. (2015). "Drug development for neurodegenerative diseases," in Neurodegenerative Disorders as Systemic Diseases, ed. K. Wada (Tokyo: Springer), 183-216. doi: 10.1007/978-4-431-54541-5_9

Nagai, Y., Tucker, T., Ren, H., Kenan, D. J., Henderson, B. S., Keene, J. D., et al. (2000). Inhibition of polyglutamine protein aggregation and cell death by novel peptides identified by phage display screening. J. Biol. Chem. 275, 10437-10442. doi: $10.1074 / \mathrm{jbc} .275 .14 .10437$

Niu, C., Prakash, T. P., Kim, A., Quach, J. L., Huryn, L. A., Yang, Y., et al. (2018). Antisense oligonucleotides targeting mutant Ataxin-7 restore visual function in a mouse model of spinocerebellar ataxia type 7. Sci. Transl. Med. 10: eaa8677.

Nucifora, L. G., Burke, K. A., Feng, X., Arbez, N., Zhu, S., Miller, J., et al. (2012). Identification of novel potentially toxic oligomers formed in vitro from mammalian-derived expanded huntingtin Exon-1 protein. J. Biol. Chem. 287, 16017-16028. doi: 10.1074/jbc.m111.252577

Okamoto, Y., Nagai, Y., Fujikake, N., Akiko Popiel, H., Yoshioka, T., Toda, T., et al. (2009). Surface plasmon resonance characterization of specific binding of polyglutamine aggregation inhibitors to the expanded polyglutamine stretch. Biochem. Biophys. Res. Commun. 378, 634-639. doi: 10.1016/j.bbrc.2008. 11.094

Olshina, M. A., Angley, L. M., Ramdzan, Y. M., Tang, J., Bailey, M. F., Hill, A. F., et al. (2010). Tracking mutant huntingtin aggregation Kinetics in cells reveals three major populations that include an invariant oligomer pool. J. Biol. Chem. 285, 21807-21816. doi: 10.1074/jbc.m109.084434

Orr, H. T., and Zoghbi, H. Y. (2007). Trinucleotide repeat disorders. Annu. Rev. Neurosci. 30, 575-621.

Pardridge, W. M. (1983). Brain metabolism: a perspective from the bloodbrain barrier. Physiol. Rev. 63, 1481-1535. doi: 10.1152/physrev.1983.63. 4.1481

Paulson, H. (2018). "Chapter 9 - Repeat expansion diseases," in Handbook of Clinical Neurology, eds D. H. Geschwind, H. L. Paulson, and C. Klein (Amsterdam: Elsevier), 105-123. doi: 10.1016/b978-0-444-63233-3. 00009-9

Poirier, M. A., Li, H., Macosko, J., Cai, S., Amzel, M., and Ross, C. A. (2002). Huntingtin spheroids and protofibrils as precursors in polyglutamine fibrilization. J. Biol. Chem. 277, 41032-41037. doi: 10.1074/jbc.m2058 09200

Pollitt, S. K., Pallos, J., Shao, J., Desai, U. A., Ma, A. A. K., Thompson, L. M., et al. (2003). A rapid cellular FRET assay of polyglutamine aggregation 
identifies a novel inhibitor. Neuron 40, 685-694. doi: 10.1016/s0896-6273(03) 00697-4

Popiel, H. A., Nagai, Y., Fujikake, N., and Toda, T. (2007). Protein transduction domain-mediated delivery of QBP1 suppresses polyglutamine-induced neurodegeneration in vivo. Mol. Ther. 15, 303-309. doi: 10.1038/sj.mt.63 00045

Popiel, H. A., Nagai, Y., Fujikake, N., and Toda, T. (2009). Delivery of the aggregate inhibitor peptide QBP1 into the mouse brain using PTDs and its therapeutic effect on polyglutamine disease mice. Neurosci. Lett. 449, 87-92. doi: 10.1016/ j.neulet.2008.06.015

Ren, H., Nagai, Y., Tucker, T., Strittmatter, W. J., and Burke, J. R. (2001). Amino acid sequence requirements of peptides that inhibit polyglutamine-protein aggregation and cell death. Biochem. Biophys. Res. Commun. 288, 703-710. doi: 10.1006/bbrc.2001.5783

Robertson, A. L., Bate, M. A., Buckle, A. M., and Bottomley, S. P. (2011). The rate of PolyQ-mediated aggregation is dramatically affected by the number and location of surrounding domains. J. Mol. Biol. 413, 879-887. doi: 10.1016/j.jmb. 2011.09.014

Rué, L., Bañez-Coronel, M., Creus-Muncunill, J., Giralt, A., Alcalá-Vida, R., Mentxaka, G., et al. (2016). Targeting CAG repeat RNAs reduces Huntington's disease phenotype independently of huntingtin levels. J. Clin. Invest. 126, 4319-4330. doi: 10.1172/jci83185

Sahashi, K., Katsuno, M., Hung, G., Adachi, H., Kondo, N., Nakatsuji, H., et al. (2015). Silencing neuronal mutant androgen receptor in a mouse model of spinal and bulbar muscular atrophy. Hum Mol Genet 24, 5985-5994. doi: 10.1093/hmg/ddv300

Sathasivam, K., Lane, A., Legleiter, J., Warley, A., Woodman, B., Finkbeiner, S., et al. (2010). Identical oligomeric and fibrillar structures captured from the brains of R6/2 and knock-in mouse models of Huntington's disease. Hum. Mol. Genet. 19, 65-78. doi: 10.1093/hmg/ddp467

Saudou, F., Finkbeiner, S., Devys, D., and Greenberg, M. E. (1998). Huntingtin acts in the nucleus to induce apoptosis but death does not correlate with the formation of intranuclear inclusions. Cell 95, 55-66. doi: 10.1016/s00928674(00)81782-1

Schiffer, N. W., Broadley, S. A., Hirschberger, T., Tavan, P., Kretzschmar, H. A., Giese, A., et al. (2007). Identification of anti-prion compounds as efficient inhibitors of polyglutamine protein aggregation in a zebrafish model. J. Biol. Chem. 282, 9195-9203. doi: 10.1074/jbc.m6078 65200

Schoch, K. M., and Miller, T. M. (2017). Antisense oligonucleotides: translation from mouse models to human neurodegenerative diseases. Neuron 94, 10561070. doi: 10.1016/j.neuron.2017.04.010

Scoles, D. R., Meera, P., Schneider, M., Paul, S., Dansithong, W., Figueroa, K. P., et al. (2017). Antisense oligonucleotide therapy for spinocerebellar ataxia type 2. Nature 544, 362-366.

Simon, R. J., Kania, R. S., Zuckermann, R. N., Huebner, V. D., Jewell, D. A., Banville, S., et al. (1992). Peptoids: a modular approach to drug discovery. PNAS 89, 9367-9371. doi: 10.1073/pnas.89.20.9367

Singh, V., Patel, K. A., Sharma, R. K., Patil, P. R., Joshi, A. S., Parihar, R., et al. (2019). Discovery of arginine ethyl ester as polyglutamine aggregation inhibitor: conformational transitioning of Huntingtin N-terminus augments aggregation suppression. ACS Chem. Neurosci. 10, 3969-3985. doi: 10.1021/acschemneuro. 9 b00167

Skene, D. J., Middleton, B., Fraser, C. K., Pennings, J. L. A., Kuchel, T. R., Rudiger, S. R., et al. (2017). Metabolic profiling of presymptomatic Huntington's disease sheep reveals novel biomarkers. Sci. Rep. 7:43030.

Snyder-Keller, A., McLear, J. A., Hathorn, T., and Messer, A. (2010). Early or latestage anti-N-terminal huntingtin intrabody gene therapy reduces pathological features in B6.HDR6/1 mice. J. Neuropathol. Exp. Neurol. 69, 1078-1085. doi: 10.1097/nen.0b013e3181f530ec

Sontag, E. M., Lotz, G. P., Agrawal, N., Tran, A., Aron, R., Yang, G., et al. (2012). Methylene blue modulates huntingtin aggregation intermediates and is protective in Huntington's disease models. J. Neurosci. 32, 11109-11119. doi: 10.1523/jneurosci.0895-12.2012

Southwell, A. L., Bugg, C. W., Kaltenbach, L. S., Dunn, D., Butland, S., Weiss, A., et al. (2011). Perturbation with intrabodies reveals that calpain cleavage is required for degradation of Huntingtin exon 1. PLoS One 6:e16676. doi: 10.1371/journal.pone.0016676
Southwell, A. L., Ko, J., and Patterson, P. H. (2009). Intrabody gene therapy ameliorates motor, cognitive, and neuropathological symptoms in multiple mouse models of Huntington's disease. J. Neurosci. 29, 13589-13602. doi: 10.1523/jneurosci.4286-09.2009

Southwell, A. L., Kordasiewicz, H. B., Langbehn, D., Skotte, N. H., Parsons, M. P., Villanueva, E. B., et al. (2018). Huntingtin suppression restores cognitive function in a mouse model of Huntington's disease. Sci. Transl. Med. 10:eaar3959. doi: 10.1126/scitranslmed.aar3959

Stanek, L. M., Yang, W., Angus, S., Sardi, P. S., Hayden, M. R., Hung, G. H., et al. (2013). Antisense oligonucleotide-mediated correction of transcriptional dysregulation is correlated with behavioral benefits in the YAC128 mouse model of Huntington's disease. J. Huntington's Dis. 2, 217-228. doi: 10.3233/jhd130057

Stoyas, C. A., and La Spada, A. R. (2018). The CAG-polyglutamine repeat diseases: a clinical, molecular, genetic, and pathophysiologic nosology. Handb. Clin. Neurol. 147, 143-170. doi: 10.1016/b978-0-444-63233-3. 00011-7

Sun, X., Marque, L. O., Cordner, Z., Pruitt, J. L., Bhat, M., Li, P. P., et al. (2014). Phosphorodiamidate morpholino oligomers suppress mutant huntingtin expression and attenuate neurotoxicity. Hum. Mol. Genet. 23, 63026317. doi: $10.1093 / \mathrm{hmg} / \mathrm{ddu} 349$

Tabrizi, S. J., Leavitt, B. R., Landwehrmeyer, G. B., Wild, E. J., Saft, C., Barker, R. A., et al. (2019). Targeting Huntingtin expression in patients with Huntington's disease. N. Engl. J. Med. 380, 2307-2316.

Takahashi, T., Kikuchi, S., Katada, S., Nagai, Y., Nishizawa, M., and Onodera, O. (2008). Soluble polyglutamine oligomers formed prior to inclusion body formation are cytotoxic. Hum. Mol. Genet. 17, 345-356. doi: 10.1093/hmg/ ddm 311

Takahashi, Y., Okamoto, Y., Popiel, H. A., Fujikake, N., Toda, T., Kinjo, M., et al. (2007). Detection of polyglutamine protein oligomers in cells by fluorescence correlation spectroscopy. J. Biol. Chem. 282, 24039-24048. doi: 10.1074/jbc. m704789200

Takeuchi, T., and Nagai, Y. (2017). Protein misfolding and aggregation as a therapeutic target for polyglutamine diseases. Brain Sci. 7:128. doi: 10.3390/ brainsci7100128

Takeuchi, T., Popiel, H., Futaki, S., Wada, K., and Nagai, Y. (2014). Peptide-based therapeutic approaches for treatment of the polyglutamine diseases. CMC 21, 2575-2582. doi: 10.2174/0929867321666140217124038

Tanaka, M., Machida, Y., Niu, S., Ikeda, T., Jana, N. R., Doi, H., et al. (2004). Trehalose alleviates polyglutamine-mediated pathology in a mouse model of Huntington disease. Nat. Med. 10, 148-154. doi: 10.1038/nm985

Tomita, K., Popiel, H. A., Nagai, Y., Toda, T., Yoshimitsu, Y., Ohno, H., et al. (2009). Structure-activity relationship study on polyglutamine binding peptide QBP1. Bioorgan. Med. Chem. 17, 1259-1263. doi: 10.1016/j.bmc.2008. 12.018

Trottier, Y., Lutz, Y., Stevanin, G., Imbert, G., Devys, D., Cancel, G., et al. (1995). Polyglutamine expansion as a pathological epitope in Huntington's disease and four dominant cerebellar ataxias. Nature 378, 403-406. doi: 10.1038/37 $8403 \mathrm{a} 0$

Verma, M., Sharma, A., Naidu, S., Bhadra, A. K., Kukreti, R., and Taneja, V. (2012). Curcumin prevents formation of polyglutamine aggregates by inhibiting Vps36, a component of the ESCRT-II complex. PLoS One 7:e42923. doi: 10. 1371/journal.pone.0042923

Wang, C.-E., Zhou, H., McGuire, J. R., Cerullo, V., Lee, B., Li, S.-H., et al. (2008). Suppression of neuropil aggregates and neurological symptoms by an intracellular antibody implicates the cytoplasmic toxicity of mutant huntingtin. J. Cell Biol. 181, 803-816. doi: 10.1083/jcb.200710158

Wang, N., Lu, X.-H., Sandoval, S. V., and Yang, X. W. (2013). An independent study of the preclinical efficacy of C2-8 in the R6/2 transgenic mouse model of Huntington's disease. J. Huntington's Dis. 2, 443-451. doi: 10.3233/jhd130074

Warrick, J. M., Paulson, H. L., Gray-Board, G. L., Bui, Q. T., Fischbeck, K. H., Pittman, R. N., et al. (1998). Expanded polyglutamine protein forms nuclear inclusions and causes neural degeneration in Drosophila. Cell 93, 939-949. doi: 10.1016/s0092-8674(00)81200-3

Welch, W. J., and Brown, C. R. (1996). Influence of molecular and chemical chaperones on protein folding. Cell Stress Chaperones 1, 109-115. doi: 10.1379/ 1466-1268(1996)001<0109:iomacc >2.3.co;2 
Wetzel, R. (2012). Physical chemistry of polyglutamine: intriguing tales of a monotonous sequence. J. Mol. Biol. 421, 466-490. doi: 10.1016/j.jmb.2012. 01.030

Wolfgang, W. J., Miller, T. W., Webster, J. M., Huston, J. S., Thompson, L. M., Marsh, J. L., et al. (2005). Suppression of Huntington's disease pathology in Drosophila by human single-chain Fv antibodies. PNAS 102, 11563-11568. doi: $10.1073 /$ pnas. 0505321102

Yang, M., Zhang, Q., Wang, Q., Sørensen, K. K., Boesen, J. T., Ma, S. Y., et al. (2018). Brain-targeting delivery of two peptidylic inhibitors for their combination therapy in transgenic polyglutamine disease mice via intranasal administration. Mol. Pharm. 15, 5781-5792. doi: 10.1021/acs.molpharmaceut.8b00938

Zhang, X., Smith, D. L., Meriin, A. B., Engemann, S., Russel, D. E., Roark, M., et al. (2005). A potent small molecule inhibits polyglutamine aggregation in Huntington's disease neurons and suppresses neurodegeneration in vivo. Proc. Natl. Acad. Sci. U.S.A. 102, 892-897. doi: 10.1073/pnas.0408936102
Conflict of Interest: YN belongs to an endowment department, supported by Nihon Medi-Physics Co., AbbVie GK., Otsuka Pharm Co., Kyowakai Med. Co., Fujiikai Med. Co., Yukioka Hosp., Osaka Gyoumeikan Hosp., Kyorin Co., and Tokuyukai Med. Co.

The remaining author declares that the research was conducted in the absence of any commercial or financial relationships that could be construed as a potential conflict of interest.

Copyright (๑) 2021 Minakawa and Nagai. This is an open-access article distributed under the terms of the Creative Commons Attribution License (CC BY). The use, distribution or reproduction in other forums is permitted, provided the original author(s) and the copyright owner(s) are credited and that the original publication in this journal is cited, in accordance with accepted academic practice. No use, distribution or reproduction is permitted which does not comply with these terms. 